\title{
Research on the Effect of Puerarin on Alleviating Sports Fatigue
}

\author{
Zhang Wei*
}

Henan University of Urban Construction, China

\begin{abstract}
This paper focus on the effects of puerarin remit sports fatigue by observeing the effects of puerarinon blood components and exercise ability of mice in swimming trained. Comparing with the time of exhaustive swimming in the swim trained group, swim trained group with puerarin significantly increase $(\mathrm{P}<0.05)$. The puerarin may reduce the increase of hemoglobin, red blood cell and platelets caused by endurance exercise, reduce the blood viscosity, prolong the time of exhaustive swimming and improve exercise ability of mice in swim trained. Through the rat swimming experiment, we can further know the effects of puerarin on alleviating sports fatigue are obviously, which plays a role in the research field of the physiological effect of puerarin.
\end{abstract}

Keywords: Exhausted exercise, puerarin, sports fatigue, rat.

\section{INTRODUCTION}

Pueraria (radixpuerariae) is the dry root of leguminous plant kudzu (puerar. Ialobata) or kudzu (puerariathansonilbenth). Chinese earliest medical monograph of cold-induced febride puerarin as in the article, and recorded the sexual ignorance and effect of radix puerariae, which has the function of muscle solution, bring down fever, promote the secretion of saliva or body fluid, promoting eruption, and it is used in the treatment of exogenous fever, headache, heat dysentery, diarrhea, measles, dizziness, etc. In the period of Han dynasty, the nourish square "pueraria decoction" was by recorded Zhang Zhongjing in treatise on febrile disease.In recent years, active research on a variety of effective components of radix puerariae, which are mainly the exploration for its pharmacological activity and metabolic characteristics.Puerarin is beginning in the cold-induced febride, as in the article, all previous dynasties materia medica are recorded, as one of the commonly used traditional Chinese medicine (TCM). Puerarin and Puerarin flavonoids, from kudzu leguminous plants or food kudzu root extract is a kind of flavone, is one of the main effective ingredients of radix puerariae, which has the effects of expansion, improving myocardial contraction of heart head blood-vessel, reduceing myocardial oxygen consumption, promoting microcirculation, and so on. In recent years, the pharmacological effects of puerarin have been extensive researched [1], to have made significant progress, now review the following for related research and application of reference.

Puerarin is one of the main effective components extracted from traditional Chinese medicine radix puerariae, which is a kind of isoflavone compounds, the previous experimental study found that puerarin has the effections of reducing inflammation, resisting oxidative damage, reducing excitability amino acid release, inhibiting the apoptosis of

*Address correspondence to this author at the Henan University of Urban Construction, China; Tel: +358-6-3247476; Fax: +358-6-3247457;

E-mail: hunter2011@foxmail.com the nerve cells [2]. And there aren't any reports at present about puerarin on the influence of sports fatigue in learning and memory and other cognitive abilities.

\section{THE PROTECTIVE MECHANISM OF PUERARIA IN SPORTS}

Free radicals refers to the molecules, atoms, ions, or groups on outer orbit contains one or more than one unpaired electrons, such as superoxide anion ( ), hydroxyl from south $(\cdot \mathrm{OH})$ and hydrogen peroxide $(\mathrm{H} 2 \mathrm{O} 2)$. Under the condition of certain pathological or strenuous exercise, when the free radicals are produced too much or remove ability descend, oxidative stress is often caused, which has damage effects on such as protein, nucleic acids, lipids, resulting in damage of body [3]such as inflammation, aging, atherosclerosis, myocardial ischemia, etc. Experiments confirmed that puerarin has the function of reducing the MDA contents of multiple organs in the body, improving the SOD content, which is a good exogenous oxygen free radical scavenger [4].

\section{PUERARIN AND MOVEMENT ABILITY}

\subsection{Puerarin can Promote Functional Recovery and Im- prove the Ability of Movement after Exercise}

Movement with great strength or a long period of time will make the dynamic balance of free radicals broken. At this time the free radical increases rapidly, oxidative stress happens, which will lead to the damage and aging of the nucleic acid, protein, fat, sugar and biological membrane. Especially when the cell membrane is attacked by free radicals, lipid peroxidation reaction is further caused to generat lipid peroxidation products. It will make obstacles of the membrane fluidity and permeability, give rise to mitochondrial damage, environment abnormal inside and outside cells, the entire cell functions drop, eventually lead to the body injury [5]. 
Puerarin can reduce lipid peroxidation of the body caused by movement, enhance the activity of antioxidant enzymes, and decrease MDA level to promote the recovery process after the movement, to play a role of the protection of the body. Zhu ql etc. [6 used a ribflavin light system to produce super oxygen anion, used Fen - ton reaction to generat hydroxyl free radical $(\cdot \mathrm{OH})$, to study the scavenging effect of PueX oxygen free radical. Results showed that Pue can eliminate $\mathrm{O} 2$ and $\cdot \mathrm{OH}$, inhibit the generatd of hemolysis of red blood cells and lipid peroxide caused by $\mathrm{H} 2 \mathrm{O} 2$, enhance SOD activity, reduce MDA content, and protect the papillary muscle from the damage of ultra oxygen anion. Exhaustion swimming trained in mice, the results found that the content of SOD of gastric mucosa in mice after endurance trained for 6 weeks in advance of immediate medication group after exhaustion exercise is increased by $16.7 \%$ more than that in the control group, the MDA content in stomach tissue of exhaustion exercise immediate medication group and recovery treatment group were obviously lower their control group, that kudzu root extract can improve the mice stomach tissue in exhaustion exercise the ability to eliminate free radicals, promote recovery after exercise mice, and have certain protection on gastric mucosa; It was also found that the trained time of the medicine group on swimming to exhaustion is significantly higher than that of the control group which further proved that puerarin has the capacity of improving exercise in mice.

\subsection{Puerarin Can Improve Energy Reserves}

Puerarin has the effect to improve energy reserves, its mechanism may be that puerarin can accelerat the exudation of insulin. $\mathrm{Xu}$ Yue through the determination the glycogen content of liver, skeletal muscle after endurance sports, found that puerarin flavonoids can obviously promote the recovery of glycogen after endurance sports, which further proves that puerarin has the function of hypoglycemic activity. Endurance exercise make skeletal muscle of sport rat to increase sensitivity on insulin, the biggest activity glycogen synthetase and hexokinase increase with the level of trained increasing, glycogen synthetase maintains high activation sensitivity which makes the synthesis of liver glycogen and muscle glycogen increased of sports medicine group sports group, on the same conditions (the same recovery time and the same diet) at the end of the movement, so that the glycogen levels of liver glycogen and muscle glycogen of sports medicine group are higher than that of exercise group. When there is a serious loss of or interrupt of coronary blood flow, significant changes appear in myocardial metabolism: compound of glucose stop oxidation, glycolysis would be the main energy source, which would reduce the ATP generation. So increasing the glycogen content of myocardial can alleviate myocardial ischemia injury, and promote resynthesis and functional recovery of myocardial glycogen when reperfusion.

\subsection{Puerarin can Reduce the Body Exercise Fatigue}

The mechanism of exercise-induced fatigue has been one of the focused research subjects by the world scholars. There are more in-depth research both at home and abroad on the exercise-induced free radical metabolism, the exercise fatigue and injuries, and sports nutrition direction. A large number of research results show that the exhaustion exercise can make the increase of endogenous free radicals generation of heart muscle, skeletal muscle, liver and other and then results in lipid peroxidation enhanced. Ding Shuzhe observed that free radical signal myocardial mitochondria of rats enhanced after exhaustion exercise with electron spin resonance instrument, and measure the MDA content increased. Tianye etc. proved the exhaustion exercise also causes mitochondrial MDA content increased, the change trend of MDA value are the same compared with exhaustion exercise, but the change rangeability below exhaustion exercise. Thus, regardless of whether they are exhaustion exercise or non exhaustion exercise can both cause the increase of generation of free radicals and it is proportional to the strength.

\subsection{Forecast}

A large number of related research for the application of puerarin provides theory basis for the applaction of puerarin function on sports nutrition food and health food. Preliminary study of the puerarin flavonoids anti-fatigue molecular mechanism also provides a theoretical basis for the application of gene chip technology using in the development and research of functional food. Because of the edible and medicinal value of puerarin, its health care function has been validated and proved to be safe and effective for a long time, with puerarin as raw material, a variety of health food has been successfully developed. Besides many pharmacological effects it has, puerarin also can supplement the body cell a variety of nutrition elements of vitality movement required, without any side effects, safety wide and rich medicine source, so it has a broad application prospect.

\section{EXPERIMENT}

\subsection{Materials and Methods}

40 healthy ICR mice, male and female half, weight 30.1 $\mathrm{g} \pm 2.5 \mathrm{~g}$, weeks age are about $11 \mathrm{w} \sim 12 \mathrm{w}$, clean level, provided by the Jinhua laboratory animal center. First male and female were randomly divided into four groups by weight: as the control group, puerarin group, swimming trained group and puerarin swimming trained group, 10 of each group, only free diet, feeding on mice IVC independent ventilation cage in the box.

\subsection{Establishment of Experimental Model}

Animal swimming pool is a white enamel barrels $(60 \mathrm{~cm}$ tall, $70 \mathrm{~cm}$ ) diameter, $50 \mathrm{~cm}$ depth, water temperature $30^{\circ} \mathrm{C}$ Group A(control group) : no swimming trained. Free diet in 3 weeks in advance, after 3 weeks, only daily injections of saline solution volume, for 3 weeks. Group B (pue control group): no swimming trained. Free diet in 3 weeks in advance, begin from 3 weeks, intraperitoneal injection puerarin ip60mg. kg-1, 3 weeks in a row. Group C (swimming trained group): adaptive trained for 1 week, up to 80 min within a week for six weeks later, swimming once a day. Group D (pue swimming trained group): swimming trained with the group C, begin from 3 weeks, injection with group B. After swimming for the last time, take blood from tail vein after $4 \mathrm{~h}$, the next day swimming exhaustion is taken. Mice of 
Table 1. Puerarin on the time of swimming to exhaustion of rats.

\begin{tabular}{|c|c|c|c|}
\hline Group & n & Exhaustion time(min) & Ratio of prolong exhaustion \\
\hline \hline Swimming controls & 20 & $844.7 \pm 124.9$ & $23 \%$ \\
\hline Puerarin+ swimming trained & 10 & $869.0 \pm 110.76$ & $28 \%$ \\
\hline
\end{tabular}

Table 2. Effect of puerarin on white blood cell of trained rat.

\begin{tabular}{|c|c|c|c|c|c|c|c|c|}
\hline & $\mathbf{n}$ & $\begin{array}{c}\text { WBC } \\
\left(\times 10^{9} / L\right)\end{array}$ & $\underset{\left(\times 10^{9} / \mathrm{L}\right)}{\text { Lym }}$ & $\begin{array}{c}\text { Mid } \\
\left(\times 10^{9} / \mathrm{L}\right)\end{array}$ & $\begin{array}{c}\text { Gran } \\
\left(\times 10^{9} / \mathrm{L}\right)\end{array}$ & $\begin{array}{c}\operatorname{Lym} \% \\
(\%)\end{array}$ & $\begin{array}{c}\text { Mid\% } \\
(\%)\end{array}$ & $\begin{array}{c}\text { Gran\% } \\
(\%)\end{array}$ \\
\hline Controls & 10 & $5.55 \pm 2.19$ & $4.09 \pm 1.83$ & $0.15 \pm 0.22$ & $1.32 \pm 0.67$ & $70.28 \pm 11.21$ & $3.95 \pm 3.29$ & $25.72 \pm 11.13$ \\
\hline Puerarin controls & 10 & $6.23 \pm 2.90$ & $3.06 \pm 1.12$ & $1.05 \pm 0.87$ & $2.10 \pm 2.02$ & $48.43 \pm 8.56$ & $18.98 \pm 13.18$ & $32.59 \pm 14.36$ \\
\hline Swimming trained & 10 & $6.81 \pm 2.37$ & $3.64 \pm 1.59$ & $0.09 \pm 0.14$ & $3.00 \pm 2.39$ & $55.08 \pm 8.33$ & $1.89 \pm 1.54$ & $43.05 \pm 17.74$ \\
\hline
\end{tabular}

Table 3. Effect of puerarin on red blood cell and hemoglobin of trained rat.

\begin{tabular}{|c|c|c|c|c|c|c|c|c|}
\hline & $\mathbf{n}$ & $\begin{array}{c}\mathbf{R B C} \\
\left(\times \mathbf{1 0}^{\mathbf{1 2}} / \mathbf{L}\right)\end{array}$ & HGB(gL) & HCT(L/L) & MCV(fL) & MCH(pg) & MCHC(g/L) & RDW\% \\
\hline \hline Controls & 10 & $9.82 \pm 1.54$ & $149.4 \pm 21.6$ & $0.463 \pm 0.086$ & $46.63 \pm 2.15$ & $15.30 \pm 0.47$ & $329.6 \pm 21.3$ & $12.58 \pm 0.92$ \\
\hline Puerarin controls & 10 & $9.66 \pm 0.82$ & $160.4 \pm 12.3$ & $0.478 \pm 0.042$ & $49.79 \pm 2.54$ & $16.30 \pm 0.87$ & $334.4 \pm 12.3$ & $12.46 \pm 0.69$ \\
\hline Swimming trained & 10 & $11.37 \pm 1.15$ & $178.3 \pm 16.5$ & $0.557 \pm 0.061$ & $49.43 \pm 2.42$ & $15.66 \pm 0.33$ & $317.6 \pm 16.2$ & $12.23 \pm 0.88$ \\
\hline $\begin{array}{c}\text { Puerarin+ } \\
\text { swimming trained }\end{array}$ & 10 & $9.80 \pm 0.72$ & $153.8 \pm 11.6$ & $0.463 \pm 0.036$ & $48.65 \pm 1.96$ & $16.20 \pm 0.47$ & $330.6 \pm 7.4$ & $12.59 \pm 0.65$ \\
\hline
\end{tabular}

exhaustion swimming groups should be put in the swimming pool at the same time. People hall drive mice in the process of swimming to prevent floating of the water. Sunk in the water when the mice were struggling, sinking to the rise of the water is seemed as exhaustion, quickly remove them, and record the discharge time.

\subsection{Blood Composition Method}

Blood cell analyzer animal model mice blood pre dilution method, 18 physiological indexes, namely WBC, Lym, Mid, Gran, Lym\%, Mid\%, Gran\%, RBC, Hb, HCT, MCV, MCH, MCHC, RDW, PLT, MPV, PCT, PDW.

\subsection{Statistical Methods}

The applacation of Excel statistical procedures for twotailed $t$ test, data is expressed by $(X+s)$.

\section{RESULTS}

\subsection{Influence of Puerarin on the Time of Swimming to Exhaustion of Rats}

Table 1 shows that through the t test, significant differences are between two groups $(\mathrm{P}<0.05)$. That expresses that puerarin could significantly increase the time of swimming to exhaustion of rats, and puerarin has significant antifatigue effect.

\subsection{Effects of Puerarin on White Blood Cells of Trained Mice}

Compared with control group, there is no difference of the total number of white blood cells between the rest three groups, but white blood cell subsets has changed, compared with control group, lymphocyte count drops, percentages of lymphocyte are significantly decreased $(\mathrm{P}<0.05)$; Blood granulocyte percentage of swimming trained group mice is increased obviously than the control group, and there is no significant difference on blood granulocyte percentage between the control group, puerarin control group and puerarin swimming trained group.

\subsection{Effect of Puerarin on RBC and Hb Trained Rat}

The Table 3 shows that the number of $\mathrm{RBC}, \mathrm{Hb}$ concentration and HCT of swimming trained group are significantly larger than the control group, and the number of RBC, concentration of $\mathrm{Hb}$ and $\mathrm{HCT}$ of puerarin control group and puerarin swimming trained has no obvious difference. The MVC and MCHC content of puerarin control group, swimming trained group and puerarin swimming trained group were significantly increased than the control group. 


\subsection{Influence of Puerarin on Platelet of Swimming Trained Mice}

The Fig. (1) shows that platelet count and platelet deposited of swimming trained group are significantly increased than the control group, while there is not significant differences between the control group and puerarin swimming trained group with platelet count and platelet deposited. There is not significant differences between the control group, swimming trained group and puerarin swimming trained group with average platelet volume and platelet distribution width. Swimming exercise trained is prompted to cause the increase of platelet count and platelet deposited, and puerarin has a regressive effect on platelet count and platelet deposited caused by swimming trained sports.

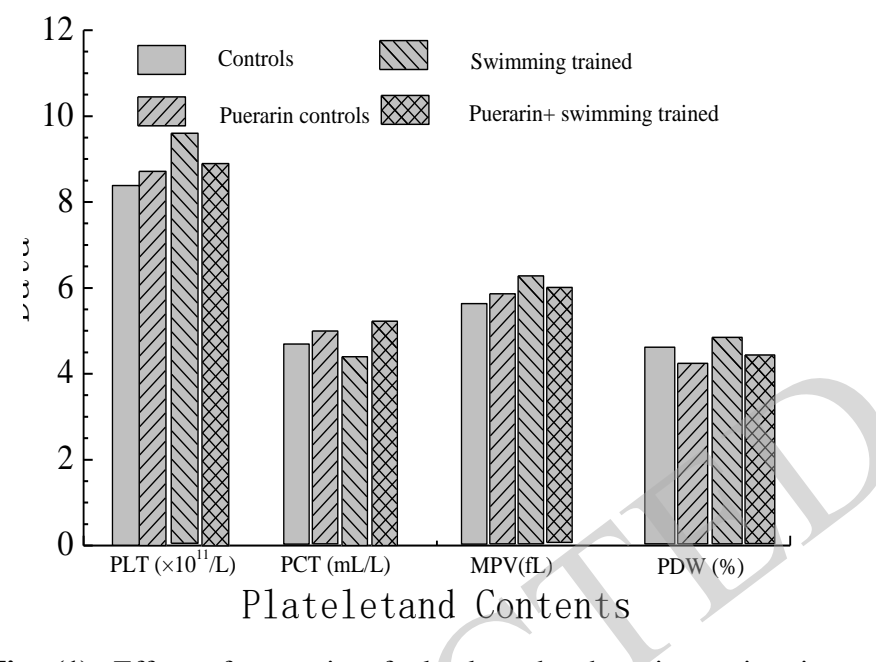

Fig. (1). Effect of puerarin of plateletand exhaustive swimming time of trained rat.

\section{CONCLUSION}

Exercise stress causes the body to produce a large number of free radicals, free radicals attack all kinds of organization to make its function decline is one of the main reasons of exercise-induced fatigue, endurance trained can improve the level of the body's antioxidant capacity and function. It is the dry root of leguminous plants field kudzu and kudzu. The influence of puerarin on the blood lipid and blood biochemical indexes exhaustion exercise rats is now reported. And provide powerful experimental basis For the further use of puerarin in the field of sports. Puerarin supplementation can reduce the level of rat whole blood viscosity, plasma viscosi- ty, erythrocyte aggregation index and erythrocyte rigidity index, effectively improve the blood rheology level, and improve the sports ability of rats. Puerarin has played a positive role on sports fatigue and recovery.

Violent movements make the body in a state of ischemia, hypoxia, oxidation, and the free radicals rise sharply, causing a chain reaction, destroying the cellular structure, resulting in lipid peroxidation, causing the body injury. Puerarin as an effective antioxidant, can reduces oxygen free radicals in the process of movement and increase the clearance rat, improve the generation of activity of SOD in the body and at the same time reducing the exercise induced increase in the number of free radicals damage of endothelial cells. Pue and sports medicine research for sports nutrition food and health food development provides a theoretical basis.

\section{CONFLICT OF INTEREST}

The authors confirm that this article content has no conflict of interest.

\section{ACKNOWLEDGEMENTS}

This work is supported by the Key Project of Guangxi Social Sciences, China (No.gxsk201424), the Education Science fund of the Education Department of Guangxi, China (No.2014JGA268), and Guangxi Office for Education Sciences Planning, China (No.2013C108).

\section{REFERENCES}

[1] Z. Chen, C. Qi, and S. Yang, "Organ aging and free radicals doctrine," Journal of Chengde Medical College, vol. 20, no.2, pp.14314, 2003.

[2] P. J. A. Rani, and C. Pannerselvam, "Carnitine as a free radical scavenger in aging," Experimental Gerontology, vol. 36, no.10, pp.1713-1726, 2001.

[3] R. Loviln, W. Cottle, I. Pyke, M. Kavanagh, and A.N. Belcastro, "Are indices of free radical damage related to exercise intensity," European Journal of Applied Physiology, vol. 56, pp.313-316, 1987.

[4] T.F. Slater, "Free-radical mechanisms in tissue injury," Journal of Biological Chemistry vol. 222, pp.1, 1984.

[5] Q. Zhu, A. He, and X. Lv, "Scavenging and resistance oxidation damage of puerarin on oxygen free radical," Chinese Journal of Polymer Science, vol. 17, no.1, pp.1-4, 2001.

[6] K. Zhang, "The effect of puerarin on SOD and MDA in gastric mucosa of rat," Chinese Journal of Sports Medicine, vol. 22, no.6, pp.614-615, 2003.

\author{
\begin{tabular}{lll}
\hline Received: May 26, 2015 & Revised: July 14, 2015 & Accepted: August 10, 2015
\end{tabular} \\ (C) Zhang Wei; Licensee Bentham Open.
}

This is an open access article licensed under the terms of the (https://creativecommons.org/licenses/by/4.0/legalcode), which permits unrestricted, noncommercial use, distribution and reproduction in any medium, provided the work is properly cited. 